\title{
Multiscale Modelling of Bubbly Systems Using Wavelet-Based Mesh Adaptation
}

\author{
Tom Liu and Phil Schwarz \\ CSIRO Minerals, Clayton, VIC 3168, Australia \\ \{Tom.Liu, Phil.Schwarz\}@CSIRO.AU
}

\begin{abstract}
Since typical industrial-scale reactors may contain many millions of bubbles, the extension of direct free-surface modelling techniques to resolve every bubble in the vessel would require far more computational power than will be available. A more immediate solution is to couple macro-scale reactor models to micro-scale models of individual bubbles and collections of a small number of bubbles. In this paper, a micro-scale modelling technique was presented and tested on the situation of a single rising bubble. The micro-scale model was based on the Volume-of-Fluid (VOF) technique combined with a dynamic mesh adaptation based on wavelet analysis to ensure a sufficient resolution at the gas-liquid interfaces. The method was based on a multi-block parallel scheme with mesh adaptivity facilitated by wavelet analysis embedded into a commercial CFD package CFX. Examples of the performance of the scheme for a bubble rising problem are given.
\end{abstract}

\section{Introduction}

Bubbly systems are used widely in the minerals and process industries to increase agitation, supply reactants (e.g. oxygen), or to effect particle separations (as in froth flotation). Effective two-fluid (or phase-averaged) modelling techniques have been developed to simulate the multi-phase fluid flow, and heat and mass transfer in such reactors, and are now widely used to assist vessel design [1,2]. These methods do not seek to resolve each bubble, but are based volume-averaged equations in which the physics of bubble-fluid interactions is modelled though constitutive terms analogous to the Reynolds stresses that appear in the RANS equations for a single phase turbulent flow. This necessarily reduces the accuracy and predictive capability of such models, and increases their dependence on empirical data and fitting parameters, particularly in cases where bubble-bubble interactions such as coalescence are important. However, in practice, it is difficult to develop accurate constitutive relations for complex systems valid over wide operating ranges using physical experiments.

A computationally efficient solution to improving the macro-scale multi-fluid models is to couple them to micro-scale models of individual bubbles and collections of a small number of bubbles. The strategy is to determine improved closure relationships for the multi-fluid model through the analysis of the micro-scale models. Where phenomena occur at widely differing scales, the numerical analysis may be employed to analyse some particular processes at fine scales, for instance, the breakup of a single bubble, 
coalescence of two or more bubbles, or bubble-particle interactions. The information obtained at fine scales can be applied in macroscale CFD or other models.

This approach has advantages over the experimental determination of constitutive relationships because physical information on the processes, e.g. shear rate dependency of the viscosity or stress field, can be obtained locally and analysed in detail. The numerical simulations used in this way may be called numerical experiments [3], though they should be viewed as the complementary to physical experiments, rather than a substitute.

The fine scale simulations differ from traditional macroscale CFD simulations used in reactor design in that more attention is paid to local information and a very fine mesh must be used to resolve full detail of interactions between the flow and the phase boundaries. If fixed meshes are employed in a traditional CFD simulation for solving practical engineering problems, the full representations of fine scale phenomena are difficult or even impossible for the foreseeable future because of the overwhelming computational costs [4]. Therefore an alternative approach that is more efficient must be developed.

Micro-scale modellings (or numerical experiments) for multiphase problems generally involve moving interfaces between immiscible fluids. The accurate simulation of fluid flows with sharp fronts presents a problem with considerable difficulties [5]. Many types of interface tracking methods have been developed, but they generally fall into two categories. In the first type, a deformable finite volume or finite element mesh is used such that a particular mesh boundary moves so that it always coincides with the interface [6]. The other strategy is to keep the mesh fixed and use a separate procedure to describe the position of the interface. These methods are reviewed in [7]. The interface can be represented on a fixed grid in a variety of ways, either explicitly or implicitly. The Volume of Fluid method (VOF) is one of the most popular implicit interface tracking schemes [5]. Physical problems which are considered in practice require three-dimensional calculations with surface tension, non-catastrophic breakage and reconnection of the interface. The VOF technique naturally allows for the latter, and has been modified by various workers to include surface tension.

Commercial software packages such as CFX and FLUENT are usually employed to model practical multiphase flows due to the complexities of the phenomena and the geometries. Although mesh adaptation has been provided by the commercial CFD packages, they generally do not allow for dynamic mesh adaptation [8]. If a mesh sufficiently fine to resolve detail near the interface is applied on the whole geometry, the computational requirements can be huge. Such a fine mesh is not necessary on most of the geometry, but because of the movement of the interface, the localized mesh refinement requires a dynamic mesh adaptation.

The largest difficulty with adaptive methods is to determine the mobility of grids. Adaptive wavelet methods have been developed to solve Navier-Stokes equation at high Reynolds numbers [9]. Wavelets have the ability to accurately and efficiently represent strongly inhomogeneous piecewise continuous functions [10]. Using such techniques, the numerical resolution can be naturally adapted to intermittent the structures of flows at fine scales with significant decrease of computational efforts and memory requirements. However, efforts have been mainly made around using wavelets as an orthogonal and complete basis, spanning a space in which to seek approximate solutions satisfying the equation in a Galerkin or collocation sense [4, 9]. To 
apply such methods however would require the development of a new program for each particular flow-related problem, whereas general-purpose commercial CFD packages are preferred.

Hestheven and Jameson [4] developed a different approach to utilise the unique properties of wavelets, and this approach can be applied in a grid-based method utilized by CFD packages. In this method, wavelets were employed to detect the existence of high frequency information and supplied spatial locations of strongly inhomogeneous regions. Very fine grids were used only in these regions. In this method, wavelets were used for grid generation and order selection only, whilst the scheme for solving the partial differential equation was based on conventional finite difference/element schemes, albeit defined on variable grids. The method provides a possibility to embed the wavelet-based grid generation into current commercial CFD packages to reduce computational costs by using adaptive meshes.

In this paper, a multi-block (domain) parallel scheme with the adaptivity facilitated by wavelet analysis was proposed and embedded into commercial CFD package CFX to track moving free surfaces efficiently.

\section{Wavelet Analysis and Wavelet-Based Grid Adaptation}

Wavelet analysis is an emerging field of applied mathematics that provides tools and algorithms suited to the type of problems encountered in multiphase process simulations. It allows one to represent a function in terms of a set of base functions, called wavelets.

Wavelet transform involves representing general functions in terms of simple, fixed building blocks at different scales and positions. These building blocks, which are actually a family of wavelets, are generated from a single fixed function called the "mother wavelet" by translation and dilation (scaling) operations. In contrast to the traditional trigonometric basis functions which have an infinite support, wavelets have a compact support. Therefore wavelets are able to approximate a function without cancellation. In the basic work of Daubechies [10], a family of compactly supported orthonormal wavelets is constructed. Each wavelet number is governed by a set of $L$ (an even integer) coefficients $\left\{p_{k}: k=0,1, \cdots, L-1\right\}$ through the two-scale relation:

$$
\phi(x)=\sum_{k=0}^{L-1} p_{k} \phi(2 x-k)
$$

and the equation:

$$
\psi(x)=\sum_{k=2-L}^{1}(-1)^{k} p_{1-k} \phi(2 x-k)
$$

where functions $\phi(x)$ and $\psi(x)$ are called scaling function and wavelet, respectively. The fundamental support of the scaling function $\phi(x)$ is in the interval $[0, L-1]$ while that of the corresponding wavelet $\psi(x)$ is in the interval $[1-L / 2, L / 2]$. The 
coefficients $p_{k}$ appearing in the two-scale relation (1) are called wavelet filter coefficients. All wavelet properties are specified through these coefficients. For more details see Daubechies' paper [10].

Interpolating functions are generated by the autocorrelation of the usual compactly supported Daubechies scaling functions [11]. Such an autocorrelation function $\theta(\cdot)$ verifies trivially the equality $\theta(n)=\delta_{0 n}$, and generates a multi-resolution analysis. The approximate solution of the problem $u_{j}(\cdot)$ defined on interval $[0,1]$ is written in terms of its values in the dyadic points:

$$
u_{j}(x)=\sum_{n} u_{j}\left(2^{-j} n\right) \theta\left(2^{j} x-n\right)
$$

and such a function is exact at the dyadic points.

Consider the function:

$$
\theta(x)=\int_{-\infty}^{+\infty} \phi(y) \phi(y-x) d y
$$

Denote $V_{j}$ the linear span of the set $\left\{\theta\left(2^{j} x-k\right), k \in Z\right\}$. It can be proven that $V_{j}$ forms a multi-resolution analysis where $\theta(\cdot)$ plays the role of a scaling function (nonorthonormal). In this paper, a modified interpolating function was constructed in order to achieve an interpolating operator on the interval with the same accuracy as the counterpart on the line. Such functions were introduced by Bertoluzza and Nald [11].

$$
\tilde{\theta}_{j 0}=\sum_{k=-L+1}^{0} \theta_{j k}, \quad \tilde{\theta}_{j, 2^{j}}=\sum_{k=2^{j}}^{2^{j}+L+1} \theta_{j k}
$$

Consider $I_{j} u$ as the form:

$$
\mathrm{I}_{j} u=u(0) \tilde{\theta}_{j 0}+\sum_{k=1}^{2^{j}-1} u\left(x_{k}\right) \theta_{j k}+u(1) \tilde{\theta}_{j 2^{j}}
$$

The sparse point representation (SPR) and the grid generation technique based on Holmstorm's work [12] were employed for grid adaptation. The SPR was based on interpolating wavelet transform (IWT) on dyadic grids. A feature of the basis is the one-to-one correspondence between point values and wavelet coefficients. The interpolating subdivision scheme recursively generated the function values on a fine grid from the given values on a coarse grid. At each level, for odd-numbered grid points, the differences between the known function values and the function values predicted by the interpolation from the coarser grid were calculated. These differences were termed as wavelet coefficients $d_{k}^{j}$, which gave the information about the irregularity of the function: $d_{k}^{j}=u\left(x_{2 k+1}^{j+1}\right)-I^{j} u\left(x_{2 k+1}^{j+1}\right)$. 


\section{Problem Definition and Method Formulation}

Wavelet-based adaptive methods have been successfully applied to solve many problems [13, 9]. The grids were adapted based on the change of the solutions. Fine meshes were assigned only on the regions where the solutions changed sharply. However, for current CFD commercial packages, e.g. CFX, there is a restriction to the dynamic mesh adaptation: the topology of the mesh must remain fixed. Therefore, the advantages of wavelet-based grid adaptation cannot be fully utilised at this stage. A modified wavelet-based mesh adaptation methodology was proposed in this section.

Wavelet-based grid generation supposes a calculation which begins with evenly spaced samples of a function. It has usually been applied to simple geometries and structured grids [11]. To combine wavelet analysis with CFX commercial CFD package, a multi-block (domain) formulation was proposed. A moving cubic subdomain or block with fine mesh was designed to track the free surface and combined the geometric flexibility and computational efficiency of a multi-block (domain) scheme with the wavelet-based mesh adaptivity. The moving block was like a "microscope" - it was used to track local information required. Coarse mesh was employed outside the moving block (subdomain). Therefore the scheme was computationally efficient without loss of accuracy.

To clearly describe the scheme, a simple case, a single rising bubble, was considered. Two algorithms were proposed as follows: wavelet-based adaptive structured grid using junction box routines; wavelet-based adaptive unstructured grid using CFX expression language and junction box routines

\subsection{Problem Definition}

A numerical experiment was designed by Krishna and Baten [14] to simulate the single bubble rising process using CFX 4 . A 2 D rectangular column $(25 \mathrm{~mm} \times 90 \mathrm{~mm})$ involving 144000 grid cells was used. It took about two weeks using six R8000 processors for a simulation run.

In this paper, the same configuration was modelled using CFX 5.7. The only difference was that a wavelet-based mesh adaptation algorithm was embedded into the CFX package so that a moving fine-mesh block was used to track the bubbles. This technique used only $10 \%$ of the number of grid cells that would be required for a uniform mesh to obtain similar accuracy.

The simulation was carried out in a rectangular column using 2D Cartesian coordinate grid. No-slip wall boundary condition was imposed, and the column was modelled as an open system. The VOF model was employed to describe transient motion of the gas and liquid phases using the Navier-Stokes equations. Surface tension was included. For the convective terms, high resolution differencing was used. First order backward Euler differencing was used for time integration. The time step used in the simulation was $0.00003 \mathrm{~s}$ or smaller.

\subsection{Wavelet-Based Adaptive Structured Grid Using Junction Box Routines}

CFX-MESHBUILD was used to generate the initial structured mesh consisting of five blocks. To utilise wavelet analysis, the proposed moving fine-mesh block must be 
either a cube or a rectangle. The initial size and position of the block depends on those of the bubble at initial time, see Table 1. Therefore the proposed mesh adaptation method can be applied in a complex geometry. The mesh distribution of block B1 was chosen as uniform grid $\left(2^{7}+1\right) \times\left(2^{6}+1\right)$ to apply wavelet analysis. Because the bubble extended in $\mathrm{x}$-direction significantly during the process, higher resolution was used in this direction. The mesh distributions of other blocks can be different. For simplicity, the same distributions were given for the shared edges and uniform grids were chosen, see Figure 1 and Table 2.

Table 1. Initial bubble/block size and position

\begin{tabular}{|c|c|}
\hline Initial Bubble (Diameter) / Block & Initial Bubble / Block Positions (Centre) \\
\hline $4 \mathrm{~mm} /(6 \mathrm{~mm} \times 6 \mathrm{~mm})$ & $(12 \mathrm{~mm}, 4 \mathrm{~mm}) /(12 \mathrm{~mm}, 4 \mathrm{~mm})$ \\
\hline $8 \mathrm{~mm} /(10 \mathrm{~mm} \times 10 \mathrm{~mm})$ & $(12 . \mathrm{mm}, 6 \mathrm{~mm}) /(12 \mathrm{~mm}, 6 \mathrm{~mm})$ \\
\hline
\end{tabular}

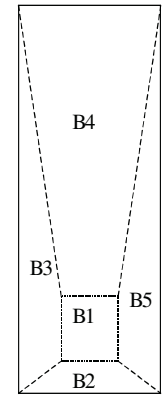

Fig. 1. Geometry and blocks of the bubble rising column
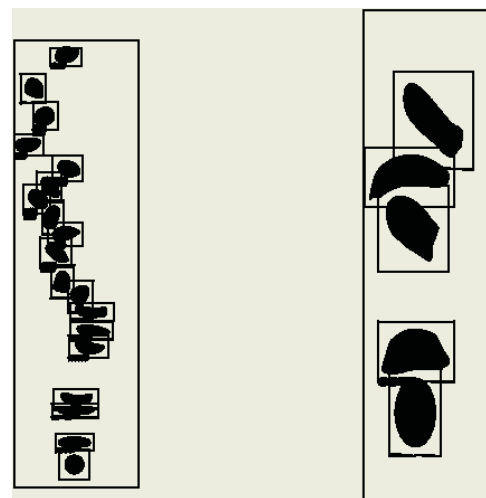

Fig. 2. Snapshots of typical rising trajectorie of bubbles of 4 and $8 \mathrm{~mm}$ diameter and $\mathrm{B} 1$

Table 2. Distributions of blocks

\begin{tabular}{|l|c|c|c|c|}
\hline & Distribution of B1 & $\begin{array}{l}\text { Distributions of B2 and } \\
\text { B4 }\end{array}$ & $\begin{array}{l}\text { Distributions of B3 and } \\
\text { B5 }\end{array}$ & $\begin{array}{l}\text { Ratio of adap- } \\
\text { tive mesh to full } \\
\text { mesh }\end{array}$ \\
\hline $4 \mathrm{~mm}$ bubble & $\left(2^{7}+1\right) \times\left(2^{6}+1\right)$ & $2^{7} \times 2^{5}$ & $2^{6} \times 2^{5}$ & 0.08 \\
\hline $8 \mathrm{~mm}$ bubble & $\left(2^{7}+1\right) \times\left(2^{6}+1\right)$ & $2^{7} \times 2^{5}$ & $2^{6} \times 2^{5}$ & 0.08 \\
\hline
\end{tabular}

Using CFX 5.7, the volume fractions on the vertices of each cell can be obtained. Due to the limitation of CFX solver, the topology of the mesh must remain fixed. Points cannot be added to or removed from the grid. Only the position of free interface was detected using IWT and wavelet coefficients. At each mesh adaptation time step, the minimum distances between the interface and the boundaries of block B1 
was calculated to decide if the position and size of B1 should be changed and a new grid was generated. The algorithm is as follows:

Step 1. Calculate the wavelet coefficients of volume fraction of gas in x-direction from the top and bottom rows of B1 to determine the nearest position between the interface and the top or bottom boundaries of B1. The IWT and wavelet coefficients are calculated from level 6 to level 7 on each row. If most of the wavelet coefficients on one row are larger than the threshold $\varepsilon$, e.g. $80 \%$ of the total number on one row, this means the interface is on or near the position.

Step 2. Calculate the wavelet coefficients of volume fraction of gas in y-direction from the left and right columns of B1 to determine the nearest position between the interface and the left or right boundaries of B1 using the same method in Step 1.

Step3. If all the distances between the interface and the boundaries of B1 are larger than given minimum distance $d_{p}$, the mesh remains fixed; Otherwise, move the boundaries of $\mathrm{B} 1$ to the positions until the distances between the interface and the boundaries are not less than $d_{p}$.

Step 4. Set limits to the position of block B1 to avoid mesh folding. There should be a given minimum distance between $\mathrm{B} 1$ and the boundaries of the column.

Step 5. Generate a new uniform mesh based on the new position of B1 for each block using the same distribution as that of the initial mesh and pass the new grid to CFX.

The block B1 was moved based on the wavelet analysis of the volume fraction function of gas. Therefore, the bubble was always in B1. There is a correlation between the frequency of the grid adaptation and the tolerance distance between the interfaces and the boundaries of B1 when the adaptation is performed. If the grid adaptation is not done often enough, a larger tolerance distance must be given so that the front does not move out of B1 between the adaptations. Conversely, more frequent adaptations demand a small distance.

It is natural to define the parallel computation partitions based on five blocks. In this work, three partitions were defined, partition 1 including B1, partition 2 including B2 and B3, partition 3 including B3 and B4. Partition 1 was calculated on master machine. The whole grid was determined based on partition 1 and passed to slave machines.

In practice, unstructured grids are often used in complex geometries. The proposed method can also be applied to unstructured grids using CFX expression language and junction box routines together. The method has been used for the same simulation as described above.

\section{Simulation Results}

The simulations were run on a Linux system. Snapshots of typical bubble trajectories are shown in Fig 2. They were similar to the results of Krishna and Baten [13]. Figure 2 clearly showed how the fine mesh moves with the bubble. It confirmed the ability to use the wavelet analysis within a multi-block framework to achieve considerable savings in computing time without loss of accuracy. The number of grid cells was 
reduced by $90 \%$. The CPU time of the adaptive mesh method was only $1 / 8$ that of the method using the fully fine mesh.

\section{Conclusions}

A wavelet-based adaptive mesh scheme was embedded into a commercial CFX package to track moving free surfaces. It significantly saves computational cost and memory requirements. The scheme was demonstrated in a single bubble rising simulation. A moving "microscope" was designed to reveal details of local information with a realistic computing time. The scheme provides the possibility to design some numerical experiments to analyse processes at a fine scale and utilise the local information obtained in macroscale models. Further work to apply the method in such a scheme is underway.

\section{References}

1. Lane, G.L, Schwarz, M.P., Evans, G.M.: Predicting gas-liquid flow in a mechanically stirred tank. Applied Mathematical Modelling 26 (2002) 223-235

2. Schwarz, M.P.: Simulation of gas injection into liquid melts. Applied Mathematical Modelling 20 (1995) 41-51

3. Ohta, M., Iwasaki, E., Obata, E., Yoshida, Y.: A numerical study of the motion of a spherical drop rising in shear-thinning fluid systems. Journal of Non-Newtonian Fluid Mechanics 116 (2003) 95-111

4. Hesthaven, J.S., Jameson, L.M.: A wavelet optimized adaptive multi-domain method. ICASE Report No.97-52, NASA Langley Research Centre, Hampton, 1997

5. Unverdi, S.O., Tryggvason, G.: A front-tracking method for viscous, incompressible multifluid follows. Journal of Computational Physics 100 (1992) 125-137

6. Drew, D.A.: Mathematical modelling of two-phase flow. Annual Review of Fluid Mechanics 15 (1983) 261-271

7. Sethian, J.A.: Level Set Methods. Cambridge University Press, Cambridge, UK 1996.

8. CFX User Guide. Release 5.7, 2004, ANSYS Canada Ltd, Waterloo, Canada

9. Vasilyev, O., Paolucci, S.: A fast adaptive wavelet collocation algorithm for multidimensional PDEs. Journal of Computational Physics 138 (1997) 16-56

10. Daubechies, I.: Orthonormal bases of compactly supported wavelets. Communication on Pure and Applied Mathematics 41 (1988) 909-996

11. Bertoluzza, S., Naldi, G.: A wavelet collocation method for the numerical solution of partial differential equations. Applied and Computational Harmonic Analysis 3 (1996) 1-9

12. Holmstrom, M.: Solving hyperbolic PDEs using interpolating wavelets. SIAM Journal on Scientific Computing 21 (1999) 405-420

13. Santos, P.C., Cruz, P., Magalhaes, F.D., Mendes, A.: 2-D wavelet-based adaptive-grid method for the resolution of PDEs. AIChE Journal 49 (2003) 706-717

14. Krishna, R., Baten, J.M.: Rise characteristics of gas bubble in a $2 \mathrm{D}$ rectangular column: VOF simulations vs experiments. Int. Commn. Heat Mass Transfer 26 (1999) 965-974 